\title{
The masking-level difference in low-noise noise
}

\author{
Joseph W. Hall III and John H. Grose \\ The Division of Otolaryngology/Head \& Neck Surgery, University of North Carolina Medical School, \\ Chapel Hill, North Carolina 27599-7070 \\ William Morris Hartmann \\ Department of Physics and Astronomy, Michigan State University, East Lansing, Michigan 48824
}

(Received 16 November 1996; revised 22 August 1997; accepted 15 January 1998)

\begin{abstract}
In experiment 1 NoSo and $\mathrm{NoS} \pi$ thresholds for a $500-\mathrm{Hz}$ pure tone were obtained in a low-fluctuation masking noise and a high-fluctuation masking noise for six normal-hearing listeners. The noise bandwidth was $10 \mathrm{~Hz}$. In agreement with previous investigations, the NoSo thresholds were lower in low-fluctuation noise than in high-fluctuation noise. For three listeners, NoS $\pi$ thresholds were similar for the two types of noise, while for the other three listeners, NoS $\pi$ thresholds were higher for low-fluctuation noise than for high-fluctuation noise. In experiment 2, the masker was created by amplitude modulating a $500-\mathrm{Hz}$ pure tone by a $0-10-\mathrm{Hz}$ low-pass noise. The degree of masker fluctuation was controlled by adjusting the average modulation depth $(100 \%, 63 \%$, $40 \%$, and $25 \%$ ). The signal was a $10-\mathrm{Hz}$-wide noise centered on $500 \mathrm{~Hz}$. Results were similar to those of experiment 1: for the NoSo conditions, signal detection improved with decreasing degree of fluctuation, and for $\operatorname{NoS} \pi$ conditions, the results were subject dependent. For three listeners, $\operatorname{NoS} \pi$ thresholds were again similar in the two types of noise, while for the other three listeners, $\operatorname{NoS} \pi$ thresholds were again higher in low-fluctuation noise than in high-fluctuation noise. The results showed that a high degree of masker fluctuation sometimes facilitates $\operatorname{NoS} \pi$ detection. It is possible that the binaural detection mechanism utilizes the relatively good signal-to-noise ratios that occur in the low power or "dip" regions of fluctuating masker waveforms. (C) 1998 Acoustical Society of America. [S0001-4966(98)01305-8]
\end{abstract}

PACS numbers: 43.66.Dc, 43.66.Pn [RHD]

\section{INTRODUCTION}

Previous experiments using low-noise (Pumplin, 1985) have investigated the effects of masker amplitude fluctuation on monaural signal detection (Hartmann and Pumplin, 1988). Conditions have contrasted detection in noise having a random phase relation among components (where noise fluctuation is generally high) with detection in noise having components whose phases are selected such that the noise fluctuation is relatively low (low-noise noise). Results (Hartmann and Pumplin, 1988) indicate that monaural signal detection is better for low-noise narrow-band noise than for random-phase narrow-band noise. Similar effects were reported by Margolis and Small (1974) for a low-fluctuation noise produced by frequency modulating a sinewave by a thermal noise. The low-noise noise result is consistent with the notion that random variation in the energy of the masker is detrimental to the detection of the signal (e.g., Bos and de Boer, 1966).

There is reason to believe that studies manipulating noise fluctuation may also be of interest in conditions of binaural unmasking. For example, it is possible that results from low- and high-fluctuation noise conditions may have bearing upon models of the masking-level difference (MLD) (Hirsh, 1948). Specifically, different binaural models may be associated with different predictions concerning the effect of masker fluctuation. In the equalization-cancellation (EC) model of Durlach (1963), the stimulus waveforms at the two ears undergo auditory filtering, level/time equalization, and subtraction. For the sake of simplicity, we will consider only NoSo and $\operatorname{NoS} \pi$ stimulation. With perfect equalization, the subtraction process would completely eliminate an No masker, and masked signal detection threshold would be similar to that obtained in quiet. However, the model assumes that there is both time and amplitude "jitter" in the process, resulting in an No noise being reduced in level rather than eliminated. In contrast, the subtraction process for the $\mathrm{S} \pi$ signal essentially results in an in-phase addition of the signal, with a consequent boost in signal level. Thus, for $\operatorname{NoS} \pi$ detection, the signal-to-noise ratio is effectively increased by the EC process. For an So condition, the subtraction process results in a reduction in signal level. However, because the same jitter parameters hold for both signal and noise, the noise and signal are reduced by similar amounts in the NoSo case. Thus, for NoSo, the signal-to-noise ratio is essentially the same at the EC input and the EC output.

In the EC model, both NoSo and $\mathrm{NoS} \pi$ detection reduce to the problem of detecting signal energy in noise. If it is supposed that the decision statistic for detection is similar for the NoSo and $\operatorname{NoS} \pi$ cases, then the effects of low-noise noise should be similar for NoSo and $\mathrm{NoS} \pi$ detection. Because power fluctuations are relatively minor in low-noise noise, both NoSo and $\operatorname{NoS} \pi$ thresholds are expected to be relatively lower than in noise with prominent power fluctuations. Because effects of low-noise noise are expected to be similar for NoSo and $\operatorname{NoS} \pi$ detection, the MLD would then be expected to be similar for low-noise noise and randomphase noise.

In contrast, the MLD is expected to be relatively smaller for low-noise noise conditions from the standpoint of crosscorrelation models (Jeffress, 1948; Colburn, 1973; Stern and Colburn, 1978; Stern and Trahiotis, 1992) of the MLD. One 
reason for this prediction is related to the fact that, in crosscorrelation models, the decision statistic for binaural detection is proposed to be radically different from that associated with monaural, or NoSo, detection. As in the EC model, monaural or NoSo detection is usually hypothesized to be based upon an analysis of stimulus energy. Thus, a relatively low NoSo threshold is predicted for a low-noise noise masker. In contrast, binaural (e.g., NoS $\pi$ ) detection is assumed to be based upon a cross-correlation process. In the physiological realization of such a process, the decision statistic is presumed to be related to a change in the activity pattern of neurons responding to different degrees of interaural delay. If it is assumed that interaural correlation will be just as high for a random-phase No noise as for a low-noise No noise, there is no reason to expect that the relatively high degree of fluctuation associated with random-phase noise should be deleterious for $\operatorname{NoS} \pi$ detection. According to this interpretation, the MLD should be smaller in low-noise noise because the NoSo threshold is expected to be lower in lownoise noise than in random-phase noise, but the $\operatorname{NoS} \pi$ threshold is not expected to be lower in low-noise noise than in random-phase noise.

A second reason that MLDs might be smaller in lownoise noise is directly related to the depth of fluctuation of the masker envelope. Masker dips (where noise power is low for short temporal epochs) are associated with a relatively high signal-to-noise ratio. There is evidence that monaural detection for a signal presented in a band of random noise does not appear to be able to benefit from the good signalto-noise ratios associated with masker dips (Buus et al., 1996). This may be because it is difficult for the auditory system to determine whether a signal occurring in a masker dip is actually a signal or simply a random fluctuation of the masker. A cross-correlation mechanism offers a potential solution to this problem in that an $S \pi$ signal occurring in an No masker dip will result in interaural correlation quite different from that associated with the masker alone. If such shortduration changes in interaural correlation are effective in cueing detection, $S \pi$ thresholds might actually be better in random-phase noise (where dips are relatively deep) than in low-noise noise (where dips are relatively shallow).

In the course of this investigation, we learned of a similar on-going study of the MLD in low-noise noise maskers, with similar results, by David Eddins and Laura Barber. The manuscripts resulting from his work and the work in our laboratory have therefore been submitted as companion papers.

\section{EXPERIMENT 1: MLD IN LOW-NOISE NOISE}

\section{A. Method}

\section{Subjects}

Subjects were six listeners with normal hearing, aged between 24 and 44 years. All had previous experience listening in MLD and CMR paradigms.

\section{Stimuli}

All noise bands were centered on $500 \mathrm{~Hz}$ and had a bandwidth of $10 \mathrm{~Hz}$. Each band was composed of 11 equal- amplitude sinusoidal components. For the high-fluctuation noise, the phase relation among the 11 components was random. For the low-noise noise, the phase relations were chosen by the low-fluctuation noise algorithm (Pumplin, 1985). For each type of noise (random-phase or low-noise noise), ten different noise files were generated. One of the ten files was selected randomly from interval to interval in order to reduce possible effects associated with a particular frozen noise sample (Hanna and Robinson, 1985). Each noise file was 4096 samples long, using a sampling rate of $4096 \mathrm{~Hz}$. Noise was delivered via a 12-bit Data Translation D/A converter. The signal was a $400-\mathrm{ms}, 500-\mathrm{Hz}$ pure tone, shaped with a 50-ms squared-cosine rise/fall. The masker was gated on (50-ms squared-cosine) $100 \mathrm{~ms}$ before the signal was gated on, and was gated off simultaneously with the signal. The masker was always interaurally in phase (No) and the signal was either interaurally in phase (So) or interaurally out of phase $(\mathrm{S} \pi)$.

\section{Masker statistics}

The average kurtosis for the ten low-noise masker waveforms was 1.655 (compared to 3.0 for Gaussian noise). Figure 1 of Hartmann and Pumplin (1988) shows that 1.655 is well outside the expected distribution for kurtosis for random-phase noises. The average crest factor for the ten low-noise noise maskers was 1.710 , compared with an expected value of 2.76 for random-phase noises (Hartmann and Pumplin, 1988, Eq. C4). In contrast with the fluctuations in signal power, the low-noise noise maskers showed no special character in their instantaneous phases. Phase variation caused by the center frequency was extracted and the residual phase variation (Hartmann, 1997, Eq. 18.6) was studied. Neither the overall phase variance nor any timedependent behavior indicated differences between low-noise noise and random-phase maskers. This analysis is relevant because the rate of change in interaural difference cues for an $\operatorname{NoS} \pi$ stimulus depends upon the changes in the instantaneous phase of the masker. The analysis suggests no obvious differences between low-noise noise and random-noise maskers in this regard.

\section{Procedure}

Thresholds were determined using a three-alternative forced choice (3AFC) three-down, one-up adaptive procedure, estimating the $79.4 \%$ detection threshold (Levitt, 1971). An initial step-size of $8 \mathrm{~dB}$ was reduced to $4 \mathrm{~dB}$ after two reversals, and further reduced to $2 \mathrm{~dB}$ after two more reversals. A threshold run was stopped after 12 reversals, and the average of the last 8 reversals was taken as the threshold for a run. Four threshold runs were averaged to compute the final threshold, unless the range of the runs was greater than $3 \mathrm{~dB}$; in that case, a fifth run was obtained and included in the average. Each trial was preceded by a $300-\mathrm{ms}$ warning light. Each interval was marked by a 400 -ms light. The interstimulus interval was $300 \mathrm{~ms}$. Visual feedback was provided after each response. The stimuli were delivered binaurally by means of Sony MDR V6 earphones. 


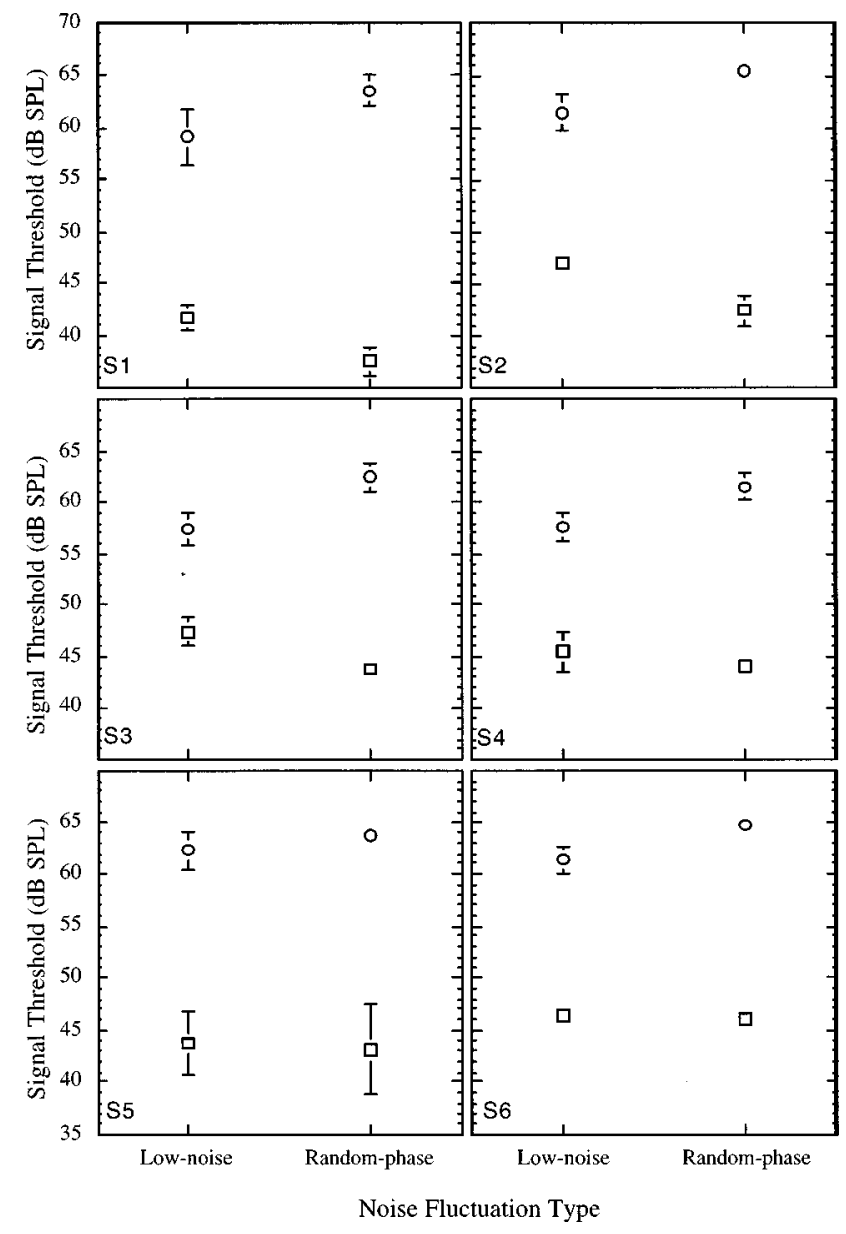

FIG. 1. The NoSo (circles) and $\operatorname{NoS} \pi$ (squares) thresholds for the six listeners, in low-noise noise (Low) and random noise (High). Error bars show plus and minus one standard deviation. Standard deviations are not shown when they are smaller than the data symbol.

\section{B. Results and discussion}

Threshold data for the six subjects are summarized in Fig. 1. Individual rather than average data are shown because there were different patterns of results across subjects. The most consistent finding, of course, was that $\operatorname{NoS} \pi$ thresholds were lower than NoSo thresholds. A second consistent finding was that, for NoSo conditions, signal thresholds were lower (by about $5 \mathrm{~dB}$ ) in low-noise noise than in randomphase noise. This result is consistent with that reported by Hartmann and Pumplin (1988). However, results for lownoise noise and random-phase noise were not consistent across subjects for $\operatorname{NoS} \pi$ detection. In NoS $\pi$ conditions, subjects 1,2, and 3 showed higher thresholds for low-noise noise than for random-phase noise. As can be seen in Table I, these subjects had considerably larger MLDs (by about 9 $\mathrm{dB}$ ) in random-phase noise than in low-noise noise. For these subjects, MLDs were relatively small in low-noise noise

TABLE I. Individual and mean MLDs ( $\mathrm{dB}$ ) for random phase noise and low-noise masking noise. Data are for experiment 1.

\begin{tabular}{lccrcccc}
\hline \hline Noise type & S1 & S2 & \multicolumn{1}{c}{ S3 } & S4 & S5 & S6 & Mean \\
\hline High fluctuation & 26.0 & 23.0 & 18.7 & 17.4 & 20.6 & 18.7 & 20.7 \\
Low fluctuation & 17.3 & 14.4 & 9.8 & 12.7 & 18.5 & 15.1 & 14.5 \\
\hline \hline
\end{tabular}

partly because the NoSo thresholds were relatively low in low-noise noise, and partly because the $\operatorname{NoS} \pi$ thresholds were relatively high in low-noise noise. For subjects 4, 5, and $6, \mathrm{NoS} \pi$ thresholds were similar between low-noise noise and random-phase noise (see Fig. 1). For these subjects, MLDs were again smaller in low-noise noise than in random-phase noise, but only by about 2 to $5 \mathrm{~dB}$. In these subjects, the smaller MLD in low-noise noise than in random-phase noise was due primarily to the fact that the NoSo thresholds were lower in low-noise noise than in random-phase noise. Across all subjects, the average MLD for random-phase noise was $20.7 \mathrm{~dB}$, and the average MLD for low-noise noise was $14.5 \mathrm{~dB}$ (see Table I).

The most striking new result of this experiment was the higher $\operatorname{NoS} \pi$ threshold in low-noise noise than in highfluctuation noise for three of the six subjects. This result suggests that a cue related to a relatively high degree of masker fluctuation can actually aid binaural signal detection. In order to examine the generality of this result, we performed a second experiment employing a different method for generating maskers with varying degrees of fluctuation.

\section{EXPERIMENT 2: MASKING NOISE CREATED BY AMPLITUDE MODULATING A PURE TONE BY A LOW- PASS NOISE}

\section{A. Method \\ 1. Subjects}

The subjects were the same as those who participated in the first experiment.

\section{Stimuli and procedure}

The masker was created by multiplying a $500-\mathrm{Hz}$ pure tone by a DC-shifted low-pass noise $(0-10 \mathrm{~Hz})$. The masking waveform was defined by

$$
A(t)=A_{0}[1+N(t)] \cos \left(\Omega_{0} t\right),
$$

where $t$ is time, $A_{0}$ is the amplitude of the $500-\mathrm{Hz}$ carrier, $N(t)$ is the low-pass noise waveform, and $\Omega_{0}$ is $2 \pi$ times the carrier frequency. A similar method was used in a MLD study by Grantham and Robinson (1977). However, in the Grantham and Robinson study, the modulator was a bandpass noise from 43 to $77 \mathrm{~Hz}$, and modulation of the $500-\mathrm{Hz}$ carrier by this bandpass noise resulted in a modulated masker with spectral components extending from approximately 423 to $577 \mathrm{~Hz}$. In the present study, the masker components were restricted to frequencies between approximately 490 and 510 $\mathrm{Hz}$. The degree of fluctuation was adjusted by varying the depth of modulation. Average modulation depths (in terms of percent of modulation) were $100 \%, 63 \%, 40 \%$, and $25 \%$, where $100 \%$ corresponds to a noise waveform $[N(t)]$ with rms value of approximately 0.707 . The level of the masker (carrier plus sidebands) was held constant at approximately $59 \mathrm{~dB}$ SPL. The signal was a $10-\mathrm{Hz}-$ wide band of noise centered on $500 \mathrm{~Hz}$. An inverse fast Fourier transform (FFT) incorporating a sampling rate of $11.025 \mathrm{kHz}$ and buffer size of $2^{17}$ discrete points was used to create the signal. This resulted in a stimulus with approximately $0.08-\mathrm{Hz}$ frequency resolution that, upon cyclical output, had an overall period- 


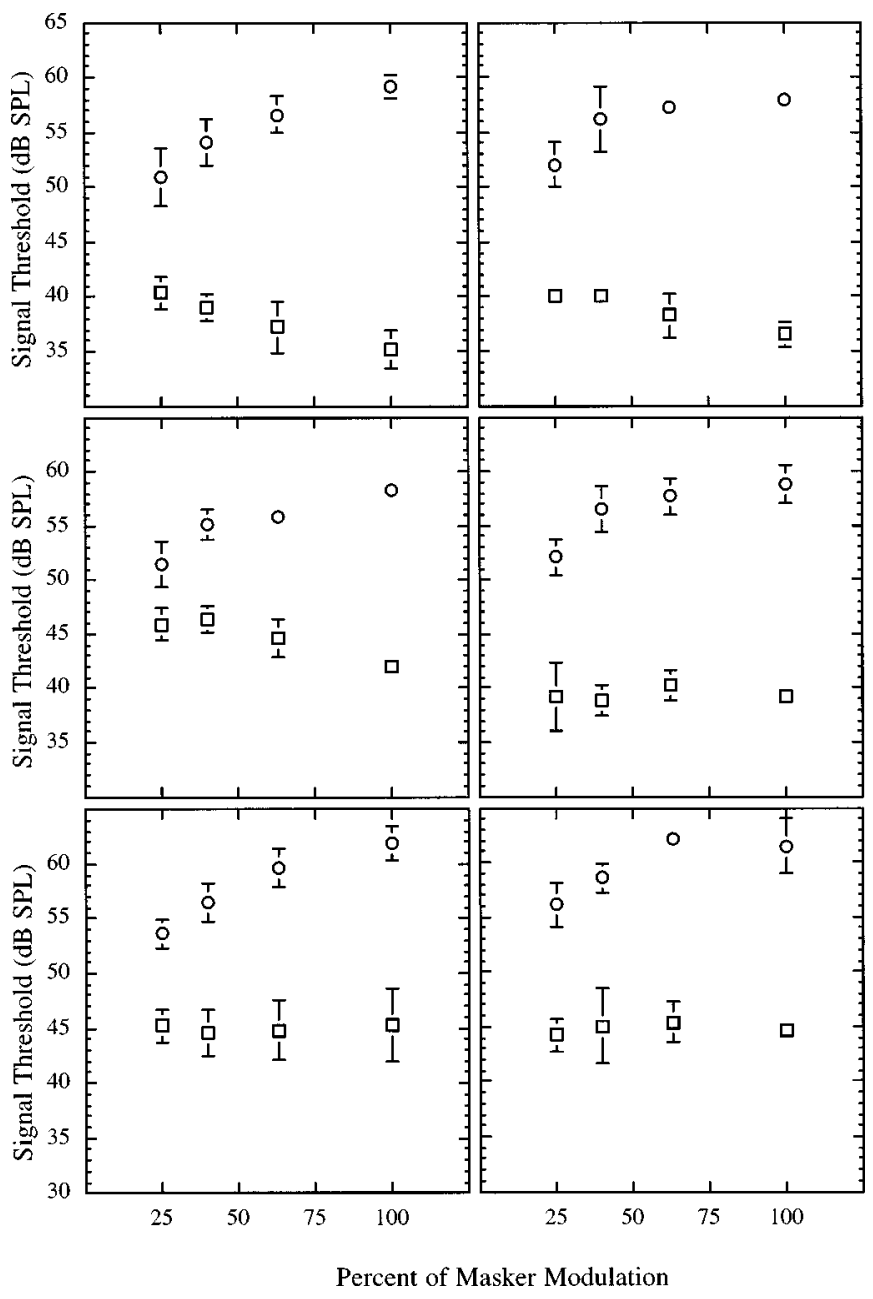

FIG. 2. The NoSo (circles) and NoS $\pi$ (squares) thresholds for the six listeners, as a function of the average percent of modulation of the masker. Error bars show plus and minus one standard deviation. Standard deviations are not shown when they are smaller than the data symbol.

icity of approximately $11.89 \mathrm{~s}$. The noise signal was played continuously through a 20-bit digital-to-analog converter and low-pass filtered at $3 \mathrm{kHz}$, and was gated via a Tucker-Davis SW2 gate. A noiseband was used as a signal, rather than a pure tone, so that the phase between the carrier of the amplitude-modulated masker would be random with respect to the phase of the signal. The signal was $400 \mathrm{~ms}$ in duration, and had 50-ms squared-cosine rise/fall. The masker was presented continuously. The masker was always interaurally in phase (No) and the signal was either interaurally in phase (So) or interaurally out of phase $(\mathrm{S} \pi)$. The threshold procedure was the same as that used in the first experiment.

\section{B. Results and discussion}

Threshold data for the six subjects are summarized in Fig. 2. In several respects, the pattern of results was similar to that obtained in experiment I. As in experiment 1, NoSo thresholds consistently improved as the masker fluctuation decreased. The average NoSo threshold was $59.6 \mathrm{~dB}$ for $100 \%$ modulation, but improved to $52.7 \mathrm{~dB}$ for $25 \%$ modulation. As in experiment 1 , there were individual differences in the effect of masker fluctuation for $\operatorname{NoS} \pi$ detection: subjects 1,2 , and 3 again showed higher thresholds with de-
TABLE II. Individual and mean MLDs for maskers varying in average percent of modulation. Data are for experiment 2 .

\begin{tabular}{cccrrrrr}
\hline \hline $\begin{array}{c}\text { Percent } \\
\text { modulation }\end{array}$ & S1 & S2 & S3 & S4 & S5 & S6 & Mean \\
\hline 100 & 23.9 & 21.4 & 16.2 & 19.7 & 16.6 & 16.9 & 19.1 \\
63 & 19.4 & 18.9 & 11.2 & 17.4 & 14.8 & 16.7 & 16.4 \\
40 & 15.0 & 16.1 & 8.7 & 17.7 & 12.0 & 13.6 & 13.8 \\
25 & 10.5 & 11.9 & 5.6 & 12.9 & 8.4 & 11.9 & 10.2 \\
\hline \hline
\end{tabular}

creases in masker fluctuation, whereas subjects 4,5 , and 6 again showed relatively stable $\operatorname{NoS} \pi$ thresholds across the different conditions of masker fluctuation. Across all subjects, the average MLDs were 19.1, 16.4, 13.8, and $10.2 \mathrm{~dB}$ for $100 \%, 63 \%, 40 \%$, and $25 \%$ modulation, respectively (see Table II). ${ }^{1}$

Again, the most striking finding was that for subjects 1, 2 , and 3, NoS $\pi$ thresholds increased as a function of decreased masker fluctuation. This result again suggests that a cue related to a relatively high degree of masker fluctuation can aid binaural signal detection. Subjects 4,5 , and 6 again showed little change in the $\operatorname{NoS} \pi$ threshold with changes in masker modulation depth.

\section{GENERAL DISCUSSION}

It was pointed out in the Introduction that a straightforward EC model interpretation would predict that NoSo and $\operatorname{NoS} \pi$ thresholds would depend similarly on masker fluctuation statistics. The findings for the $\mathrm{S} \pi$ thresholds did not agree with this prediction. The present findings were in better agreement with a cross-correlation mechanism. For a cross-correlation mechanism, it was predicted that detection for $\operatorname{NoS} \pi$ would would either not vary as a function of the degree of masker fluctuation, or that $\mathrm{S} \pi$ detection might be worse in low-fluctuation noise than in high-fluctuation noise. In three of the listeners tested, $\mathrm{S} \pi$ thresholds were similar in low-fluctuation noise and in high-fluctuation noise, and in the three other listeners, $\operatorname{NoS} \pi$ thresholds were lower in high-fluctuation noise than in low-fluctuation noise. The latter result is in direct contrast to the situation for monaural detection, where high noise fluctuation results in relatively poor detection (Bos and de Boer, 1966). It would appear that, at least in some listeners, a high degree of noise fluctuation is favorable for signal detection. It seems likely that this effect is related to low-energy or "dip" regions in the masker. In a masker having a relatively low degree of fluctuation, the signal-to-noise ratio is relatively constant over the duration of a signal. However, in a masker having a relatively high degree of fluctuation, the signal-to-noise ratio will be relatively poor in masker peak regions, but will be relatively good in masker dip regions. It is possible that the relatively short but large binaural cues that exist during masker dips are effective in cuing $\operatorname{NoS} \pi$ detection.

The above interpretation is consistent with Isabelle's (1995) account of $\operatorname{NoS} \pi$ data in a frozen noise experiment. As in previous experiments by Gilkey and his colleagues (Gilkey et al., 1985; Gilkey and Robinson, 1986), Isabelle attempted to obtain information about the cues accounting for binaural detection by examining performance for particu- 
lar digitized samples of noise alone and signal-plus-noise. While Isabelle found that $\operatorname{NoS} \pi$ performance was not correlated significantly with stimulus energy, modest, albeit significant, correlations were found for some decision statistics based upon variability of the interaural time and/or interaural intensity cues. Interestingly, the highest correlations were found for instantaneous interaural time differences. Isabelle suggested that the auditory system may utilize the infrequent but large interaural time differences occurring near the minima of the masker envelope, noting that "this strategy may be likened to 'listening in the valleys of the noise.',

One feature of binaural analysis that might be seen as in conflict with the notion of relatively fine temporal resolution has been termed "binaural sluggishness" (Grantham and Wightman, 1979). This term refers to the fact that listeners are relatively insensitive to dynamic variation in interaural difference cues, in that changes in binaural cues occurring at rates of more than a few $\mathrm{Hz}$ are not heard as movement in perceived location, but, instead as a "blur" or as diffuseness of location (Blauert, 1972; Grantham and Wightman, 1978). Grantham and Wightman (1979) reported data consistent with an interpretation that binaural sluggishness may be associated with small MLDs. They investigated the detectability of a brief $\mathrm{S} \pi$ tone burst presented in a noise masker whose interaural phase varied sinusoidally between 1.0 and -1.0 . When the short signal was presented at a time when the masker had interaural correlation near 1.0, a MLD occurred, provided that the sinusoidal modulation of interaural masker phase was very slow (e.g., $0.5 \mathrm{~Hz}$ ). However, essentially no MLD occurred when the modulation rate was raised to only $4 \mathrm{~Hz}$. This result is consistent with an interpretation that binaural sluggishness prevented the auditory system from taking advantage of the short temporal epochs when the stimulus was in NoS $\pi$ configuration. However, it reasonable to assume that the use of binaural signal detection information during short temporal epochs is poor only when both the masker and signal-plus-masker contain dynamically varying interaural cues (as was the case in the Grantham and Wightman study). When the masker has a stable interaural phase, it is likely that the binaural system can take advantage of binaural detection information in short temporal epochs. Indeed, the robust MLDs that occur for short $\mathrm{S} \pi$ signals in stable No maskers (Blodgett et al., 1958; Green, 1966; Robinson and Trahiotis, 1972; Grantham and Wightman, 1979) provide strong evidence that the binaural system can make good use of binaural detection information occurring in a short temporal epoch. We therefore do not view binaural sluggishness as incompatible with a detection process involving relatively fast sampling of interaural correlation.

\section{ACKNOWLEDGMENTS}

Constantine Trahiotis and Huanping Dai made several helpful suggestions concerning the interpretation of the results. We thank Steve Colburn for helpful suggestions and for bringing Scott Isabelle's dissertation to out attention. Raymond Dye and two anonymous reviewers made helpful suggestions on a previous version of this manuscript. We appreciate technical assistance provided by Saju Joy. This research was supported by grants from NIH (NIDCD R01 DC00397 and NIDCD R01 DC00181) and AFOSR.

${ }^{1}$ It should be mentioned that during the review process for this manuscript Kohlrausch et al. (1997) published results comparing MLD data for random-phase and low-fluctuation noise. They used a $1000-\mathrm{Hz}$ center frequency and examined masking bandwidths from 5 to $100 \mathrm{~Hz}$. For the NoSo conditions, their average data (four subjects) indicated lower thresholds for low-fluctuation noise, and for the $\operatorname{NoS} \pi$ conditions, their average data indicated similar thresholds between random-phase and low-fluctuation noise (similar to our data for subjects 4, 5, and 6).

Blauert, J. (1972). " On the lag of lateralization caused by interaural time and intensity differences,' Audiology 11, 265-270.

Blodgett, H. C., Jeffress, L. A., and Taylor, R. W. (1958). "Relation of masked threshold to signal-duration for various interaural phasecombinations,', Am. J. Psychol. 71, 283-290.

Bos, C. E., and de Boer, E. (1966). "Masking and discrimination," J. Acoust. Soc. Am. 39, 708-715.

Buus, S., Zhang, L., and Florentine, M. (1996). "'Stimulus-driven, timevarying weights for Comodulation Masking Release,', J. Acoust. Soc. Am. 99, 2288-2297.

Colburn, H. S. (1973). "Theory of binaural interaction based on auditory nerve data. I. General strategy and preliminary results on interaural discrimination,' J. Acoust. Soc. Am. 54, 1458-1470.

Durlach, N. I. (1963). "Equalization and cancellation theory of binaural masking-level differences,', J. Acoust. Soc. Am. 35, 1206-1218.

Gilkey, R. H., and Robinson, D. E. (1986). "Models of auditory masking: a molecular psychophysical approach," J. Acoust. Soc. Am. 79, 14991510

Gilkey, R. H., Robinson, D. E., and Hanna, T. E. (1985). ' Effects of masker waveform and signal-to-masker phase relation on diotic and dichotic masking by reproducible noise,', J. Acoust. Soc. Am. 78, 1207-1219.

Grantham, D. W., and Robinson, D. E. (1977). "Role of dynamic cues in monaural and binaural signal detection,'” J. Acoust. Soc. Am. 61, 542551.

Grantham, D. W., and Wightman, F. L. (1979). "'Detectability of a pulsed tone in the presence of a masker with time-varying interaural correlation," J. Acoust. Soc. Am. 65, 1509-1517.

Grantham, D. W., and Wightman, F. L. (1978). "Detectability of varying interaural temporal differences,'” J. Acoust. Soc. Am. 63, 511-523.

Green, D. M. (1966). "Interaural phase effects in the masking of signals of different durations,' J. Acoust. Soc. Am. 39, 720-724.

Hanna, T. E., and Robinson, D. E. (1985). "Phase effects for a sine wave masked by reproducible noise," J. Acoust. Soc. Am. 77, 1129-1140.

Hartmann, W. M. (1997). Signals, Sound, and Sensation (AIP, SpringerVerlag, New York).

Hartmann, W. M., and Pumplin, J. (1988). "Noise power fluctuations and the masking sine signals,'” J. Acoust. Soc. Am. 83, 2277-2289.

Hirsh, I. J. (1948). "Influence of interaural phase on interaural summation and inhibition,'” J. Acoust. Soc. Am. 20, 536-544.

Isabelle, S. (1995). "Binaural detection performance using reproducible stimuli," Ph.D. dissertation, Boston University.

Jeffress, L. A. (1948). "A place theory of sound localization," J. Comp. Physiol. Psychol. 41, 35-39.

Kohlrausch, A., Fassel, R., van der Heijden, M., Kortekaas, S., van der Par, S., and Oxenham, A. J. (1997). "Detection of tones in low-noise noise: Further evidence for the role of envelope fluctuations,' Acustica 83, 659669.

Levitt, H. (1971). "Transformed up-down methods in psychoacoustics," J. Acoust. Soc. Am. 49, 467-477.

Margolis, R. H., and Small, Jr., A. M. (1974). "'Masking with narrow-band FM noise,', J. Acoust. Soc. Am. 56, 692-694.

Pumplin, J. (1985). “'Low-noise noise,'” J. Acoust. Soc. Am. 78, 100-104. Robinson, D. E., and Trahiotis, C. (1972). "Effects of signal duration and masker duration on detectability under diotic and dichotic listening conditions," Percept. Psychophys. 12, 333-334.

Stern, R., and Colburn, H. (1978). "Theory of binaural interaction based on auditory-nerve data IV,'” J. Acoust. Soc. Am. 64, 127-140.

Stern, R. M., and Trahiotis, C. (1992). "'The role of consistency of interaural timing over frequency in binaural lateralization,', in Auditory Physiology and Perception, edited by Y. Cazals, K. Horner, and L. Demany (Pergamon, Oxford). 